\title{
Research on Organization and Optimization for Pedestrians Crossing Street Intersection in Cities Based on Vissim
}

\author{
Congcong $\mathrm{Fu}$ \\ College of Transport \& Communications \\ Shanghai Maritime University \\ Shanghai, China 201306
}

\begin{abstract}
-in order to improve the traffic capacity of pedestrian and driving at the intersection and the pedestrian safety, after the field investigation and related data analysis, this paper takes the four-phase intersection of Wuning Road and Changshou Road in Shanghai as an example to study the characteristics of pedestrian crossing traffic and its conflict with the right turning cars from east exit, propose to establish a pedestrian twice crossing facility, and adjust the signal phase setting and timing. Finally, the simulation with VISSIM shows that the setting of the twice crossing facilities can effectively improve the pedestrian capacity, safety and convenience of the intersection, reduce the pedestrian crossing delay for cars and the conflict between the two sides.
\end{abstract} phase

Keywords-intersection; twice crossing; pedestrian; signal

\section{INTRODUCTION}

Urban environmental change and economic prosperity are closely related to the gathering and dispersion of human travel. In this process, walking plays a decisive role in short-distance travel and the connection of various modes of transportation in long-distance travel. At the same time, pedestrians often collide with an increasing number of motor vehicles at the intersection of the city, causing delays between the two parties. Unreasonable phase configuration and timing, imperfect traffic organization and design will lead to pedestrian inconvenience, negative violations of street crossings, poor crossings and obstacles or conflicts with motor vehicles, and may even cause different levels of traffic accident. The traditional one-time crossing method cannot reasonably allocate the space-time resources required by pedestrians and motor vehicles, and therefore cannot reduce the conflict between the two sides. Under the effective premise, the twice crossing is set up, that is, the pedestrian safety island is set in the road, so that pedestrians who fail to cross the street by one time can have a safe and transient waiting area and cross the street twice, which reduces pedestrian violation rate and improves street crossing safety, and meanwhile, motor vehicle delays caused by obstacles in pedestrian crossings at the end of the period will be reduced. Scholars at home and abroad have done a lot of effective work in the aspects of pedestrian crossing characteristics and pedestrian twice crossing problems, which is worth learning. In 1903, William Eno, the father of traffic safety, first proposed the "safety island" in the "Traffic Rules", which has been used until now [1]. In 1977, Lalani set up safe islands in the center of 120 streets. After studying the traffic accident data for about 1 year, it is pointed out that setting up a safe island does reduce the incidence of traffic accidents [2]. In 1987, Knoblanch and Crigler analyzed the traffic characteristics of the safe island and found that the twice crossing method can shorten the distance of one-time crossing, and the safe island provides a waiting area for pedestrians to stop in the middle [3]. Domestic research on this aspect was late, but the results were quite fruitful. Yang Xiaofang and Yang Xiaoguang analyzed the twice crossings of pedestrians with signal-controlled road sections from the perspective of time-space resource utilization and delays. It was concluded that twice crossings can make more effective use of road time and space resources than one-time crossing, while the waiting time of pedestrians required for the crossing right is significantly reduced [4]. Yang Xiaoguang et al. proposed a pedestrian delay model for signal-free control sections, quantitatively compared and analyzed the pedestrian delays of the one-time and twice crossing modes under different road width and flow conditions, and finally gave the applicable range of two-way two-lane and four-lane crossing modes. [5]. By analyzing pedestrian traffic characteristics and space-time requirements, combined with reasonable signal timing and pedestrian phase method of "phase stacking", Yang Rui constructed a delay model of pedestrian twice crossing that comprehensively considers vehicle conflict and signal control, which helps to get relatively accurate delay calculation result.

Based on the current research, this paper takes the intersection of Changshou Road and Wuning Road in Shanghai as an example to analyze the current situation of intersections, including phase timing design, pedestrian crossing characteristics, traffic phenomena between pedestrians and motor vehicles. Then, the necessity of pedestrians twice crossing will be analyzed and the targeted improvement methods will be proposed. 


\section{INVESTIGATION AND ANALYSIS}

\section{A. Investigation on Current Traffic Status}

\section{1) Current road status}

The intersection is formed at Changshou Road and Wuning Road and Wuning South Road in Putuo District, Shanghai, as shown in "Fig.1". There are four roads in Wuning Road in the north, of which the center is separated by fences, the motor vehicles and non-motor vehicle lanes are separated by buffer belts, without physical facilities for separation. In the middle of Wuning South Road in the south, green plants are used to separate the motor vehicle flow from non-motor vehicle flow, without physical facility for separation. The Changshou Road of southwest-northeast direction has four lanes, of which fence isolation belt is provided at the western section, with fence installed on both sides to isolate motor vehicles and nonmotor vehicles; green plant isolation belts are installed in the middle of the eastern section, with fence installed on both sides to isolate motor vehicles and non-motor vehicles. The key parameters of entrance and exit lanes at the intersection are shown in "Table I".

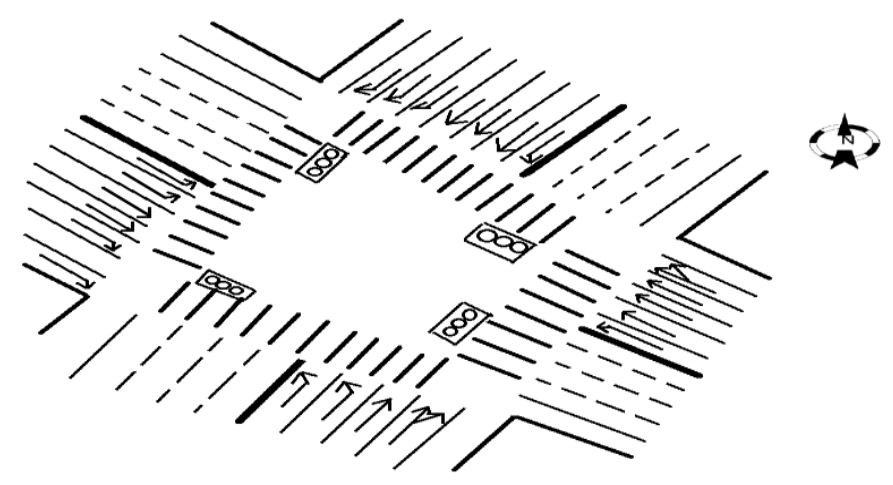

Fig. 1. Intersection diagram.

TABLE I. KEY PARAMETERS OF ENTRANCE AND EXIT LANES AT THE INTERSECTION

\begin{tabular}{|c|c|c|c|c|c|c|c|c|}
\hline Direction & \multicolumn{2}{|c|}{ East } & \multicolumn{2}{|c|}{ West } & \multicolumn{2}{|c|}{ South } & \multicolumn{2}{|c|}{ North } \\
\hline Entrance (exit) lanes & $\overline{E x i t}$ & Exit & Entrance & Exit & Entrance & Exit & Entrance & $\overline{\text { Exit }}$ \\
\hline Number of motor vehicle lanes/pc & 4 & 7 & 4 & 4 & 3 & 5 & 5 & 7 \\
\hline Non-motor vehicle lane width / m & 2.0 & 2.1 & 2.1 & 2.1 & 2.4 & 2.2 & 2.5 & 2.5 \\
\hline Motor vehicle lane width / $\mathrm{m}$ & 3.5 & 3.5 & 3.5 & 3.5 & 3.5 & 3.5 & 3.5 & 3.5 \\
\hline Isolation belt / green belt width / $\mathrm{m}$ & 1.1 & & 0.2 & & 1.1 & & 0.3 & \\
\hline Buffer band width / $\mathrm{m}$ & 0 & 0 & 0 & 0 & $\overline{0}$ & 0 & 2.1 & 2.1 \\
\hline
\end{tabular}

2) Current status of phase and timing of signal lights at The intersection is provided with a four-phase design, and the intersection its signal period is as shown in "Fig. 2".

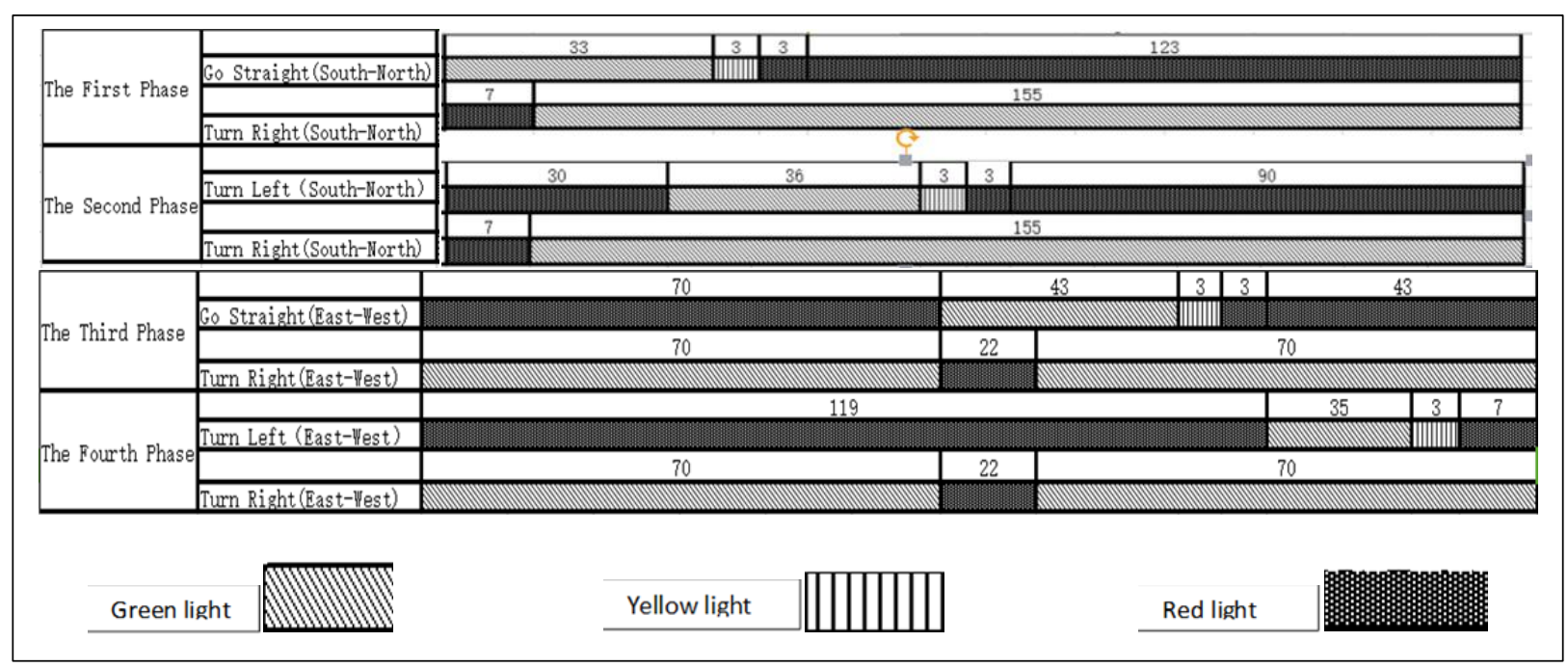

Fig. 2. The intersection is provided with a four-phase design.

\section{3)Pedestrian crossing traffic}

In "Fig. 3", "Fig. 4", "Fig. 5", "Fig. 6", Fig. 7", the complete crossing means that the pedestrian can complete the entire crossing process in the green time. The incomplete crossing means that the pedestrian cannot complete the crossing in a green light period and needs to continue to occupy the red light time.

\section{B. Traffic Analysis of Pedestrian Crossing}

Based on the above survey data, the basic situation of pedestrian traffic at the intersection is as follows: 


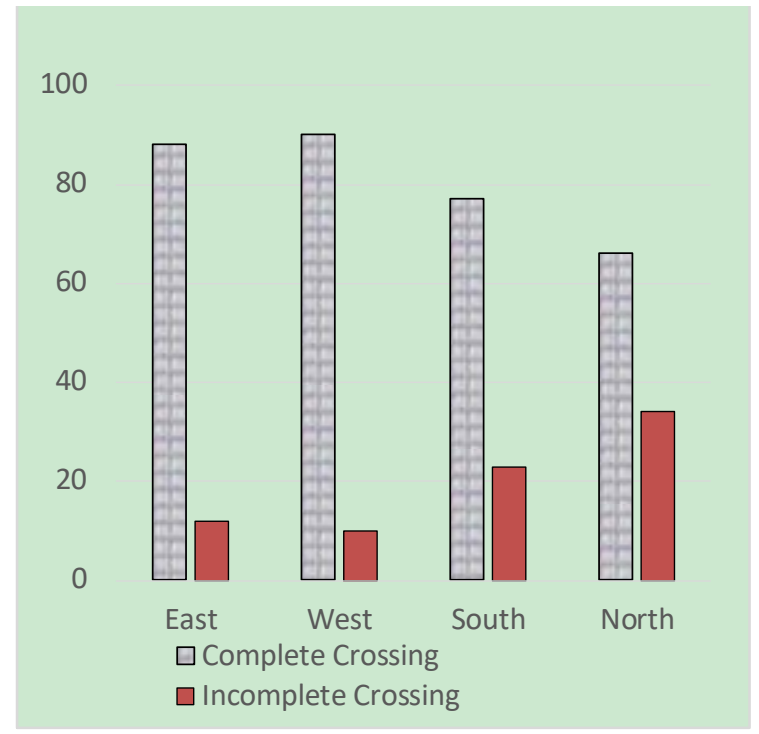

Fig. 3. Pedestrian crossing flow Proportion in different directions.

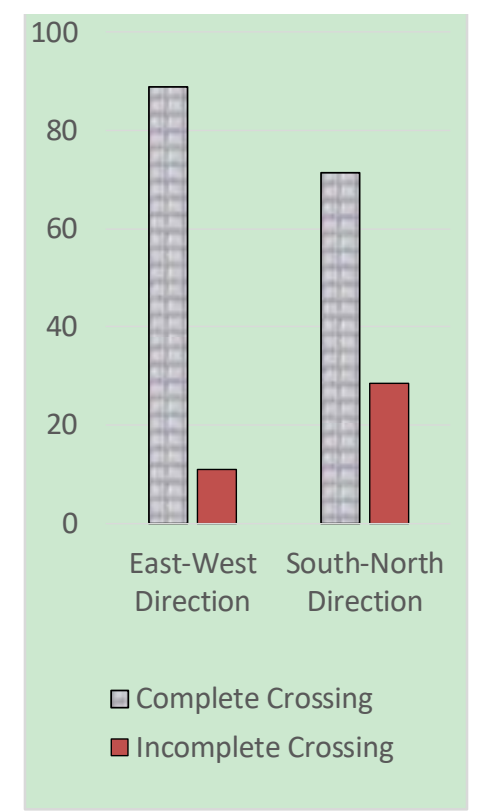

Fig. 4. Pedestrian crossing flow Proportion of in different walking directions.

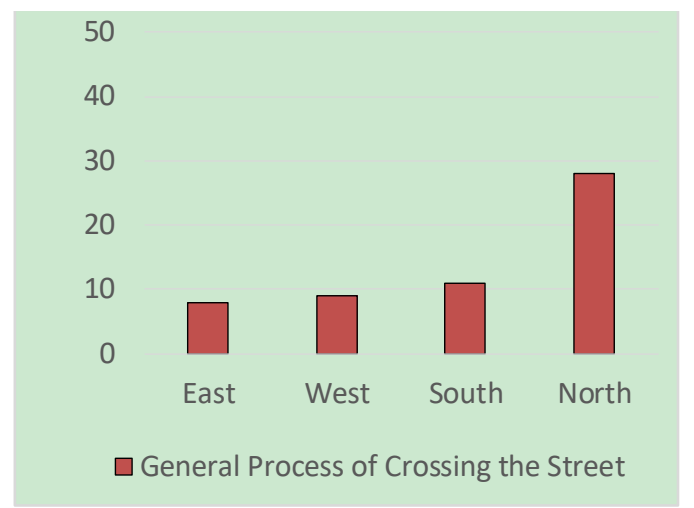

Fig. 5. Pedestrian crossing flow Proportion in different directions that have obstruction for right-turn vehicles.

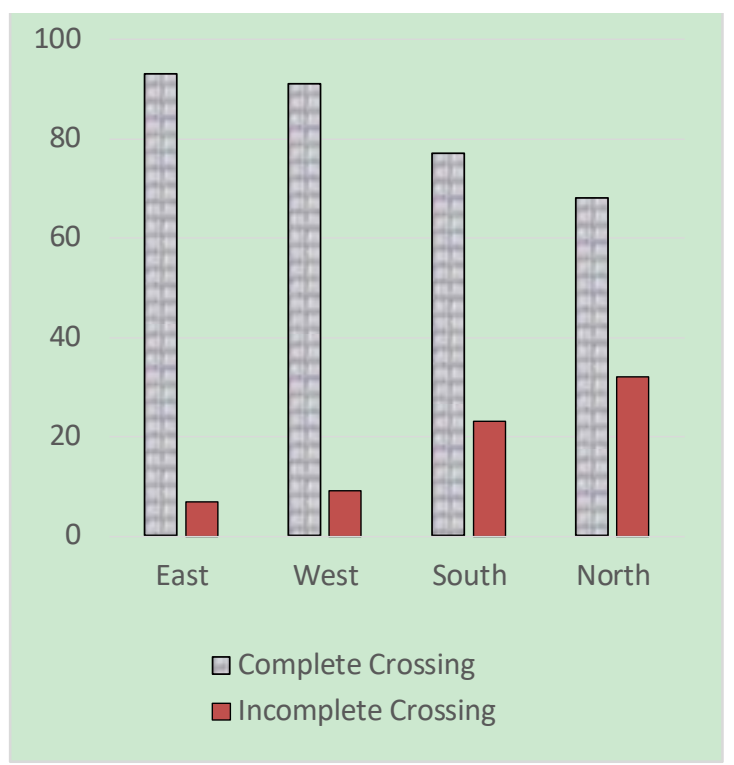

Fig. 6. Pedestrian crossing flow Proportion in different directions at the flat peak period.

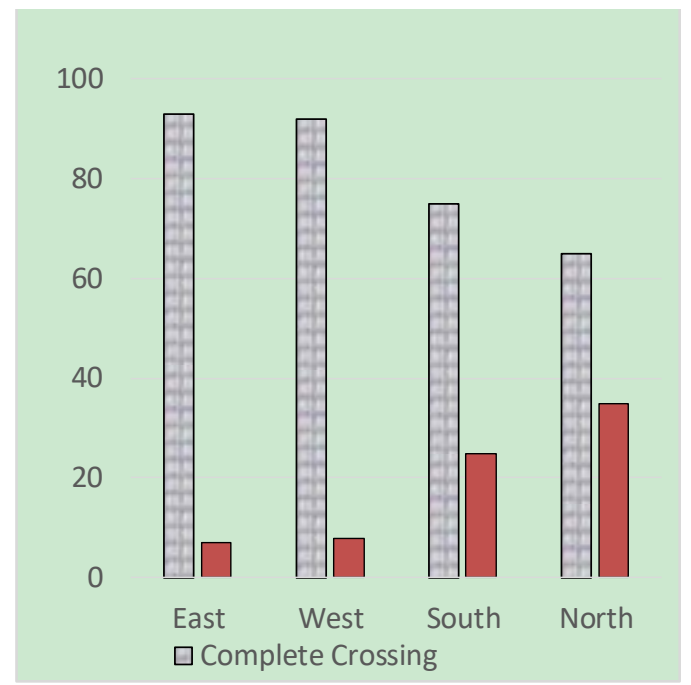

Fig. 7. Pedestrian crossing flow Proportion in different directions at the high peak period.

At the east side of Wuning Road, there is the entrance and exit of Wuning Road subway station, where there are food plaza, bank, entertainment venues and other attractions nearby, and the continuous pedestrians leave or enter the station. This section of crosswalk is long, and the full passing rate is only $66 \%$. After field investigation, the full crossing behavior of pedestrian starting from initial green light is completed within 20 seconds after the end of the green light, and the pedestrian who starts to cross the street in the middle of the green light takes longer. At the end of the green light, about 30\% of the pedestrians have not completed the crossing, while the rightturning vehicles at the eastern section of Changshou Road have started to drive, so the pedestrian crossing has obstruction on the right-turning vehicles and the pedestrian crossing flow proportion is up to $28 \%$. Pedestrians are looking for gap for crossing, with very low safety, and the vehicle avoids pedestrians, causing delays and a certain length of queue. The 
reason for the result is a long road section, large pedestrian flow, long pedestrian queue, strong mutual interference, and short green light time, which is far from meeting the time and space requirements of the crossing.

The pedestrians crossing flow is less at the Wuning South Road that at the Wuning Road. The pedestrian crossing is short, and the full passing rate of pedestrians is $77 \%$. The difference of incomplete passing rates between at flat peak and at high peak is not large, which is $23 \%$ and $25 \%$ respectively. The main reason is that the green light duration is not designed reasonably based on current pedestrian flow. In the following, the improvement plan will be given for timing of the signal light.

The east-west pedestrian crossing rate is very high, and there is little difference between at the flat peak and at the high peak, both about $90 \%$. There are few obstacles to the rightturning vehicles, and the phase is reasonable, so it doesn't need an improvement.

\section{IMPROVEMENT SCHEME}

\section{A. The Necessity of Pedestrian Twice Crossing}

From the above analysis, it can be seen that the passing rate of pedestrian walking towards south or north is high during the green light period, whether it is in usual time or traffic peak. The complete crossing ratio of pedestrian walking towards east or west is only about 70\%, especially in Wuning segment, and the incomplete crossing ratio exceeds $30 \%$ in usual time and traffic peak. On this basis, the twice crossing will not be set up on the north-south road, while Wuning segment will be equipped with a twice crossing in the form of safety island. For segment width reasons, a twice crossing will not be temporarily considered in South Wuning segment.

In terms of the road, Design Regulations for At-grade Intersections on Urban Street [7] in Shanghai takes the number of lanes as the criterion: when the number of motorway at the entrance and exit is greater than or equal to 6 , we can consider to set up the safety island by using the form of twice crossing. The number of motor vehicle entrance and exit lanes in Wuning segment is 11 , the twice crossing facilities are necessary.

In terms of the pedestrian waiting time, pedestrian waiting time in Wuning segment is more than $60 \mathrm{~s}$, which exceeds pedestrian's tolerable time limit of 50s. Table 2 shows the research results of domestic and foreign scholars on the tolerable waiting time for pedestrians crossing the street. Table 2 shows the results of a study on tolerable waiting time of pedestrian crossing carried out by domestic and foreign scholars. Due to the unreasonable signal configuration and imperfect crossing facilities, some pedestrians cross the rightturn lane and non-motor vehicle lane at red time and intend to cross the street ahead of time, which causes serious pedestrian and road safety problems. Therefore, the twice crossing facilities should be considered.

Based on the above analysis for Wuning segment, it is necessary to set up twice crossing facilities.

TABLE II. Domestic And Foreign Study of Tolerable Waiting Time of Pedestrian CRossing

\begin{tabular}{llccc}
\hline $\begin{array}{l}\text { Scholar } \\
\text { (Country) }\end{array}$ & $\begin{array}{l}\text { N Rouphail } \\
\text { (England) }\end{array}$ & $\begin{array}{c}\text { Capacity Manual } \\
\text { (Germany) }\end{array}$ & $\begin{array}{c}\text { Asaba\&Satio } \\
\text { (Japan) }\end{array}$ & $\begin{array}{l}\text { Martin\&Johnson } \\
\text { (North America, Europe) }\end{array}$ \\
\hline Tolerable Waiting Time(s) & $45 \sim 60$ & 60 & $40 \sim 45$ & 40 \\
\hline
\end{tabular}

\section{B. Improvement Scheme and Design for Pedestrian Twice Crossing}

\section{1) Improvement scheme for pedestrian twice crossing}

Based on the above analysis, it is proposed to set up a safety island in Wuning segment to organize pedestrians to complete the twice crossing. Code for Transport Planning on Urban Road [10] points out that the width of safety island should be $1.5-2.0 \mathrm{~m}$ under normal conditions, but it can be increased to $2.0-3.0 \mathrm{~m}$ in the case of a small number of nonmotor vehicles. Therefore, the width of the integrated pedestrian safety island should be 2.0 - 3.0m. Shanghai Construction Standard - Design Regulations for At-grade Intersections on Urban Street [11] stipulates that the minimum width of a motor vehicle lane at a governance intersection should be $2.75 \mathrm{~m}$ when the land area is limited.

The improvement scheme for Wuning segment: the buffer strips on both sides of non-motor vehicle of entrance and exit lanes, and between motor vehicles are compressed by $0.5 \mathrm{~m}$ respectively, that is, $2 \mathrm{~m}$ in total. An entrance lane and an exit lane on both sides of intermediate isolation strip are compressed by $0.5 \mathrm{~m}$ respectively, that is, $1 \mathrm{~m}$ in total. The fence-typed isolation strip in the middle is changed as marking, that is, to be compressed by $0.2 \mathrm{~m}$. In this way, the width provided to set up the safety island is $3.2 \mathrm{~m}$.

\section{2) Setting of pedestrian's twice crossing facilities}

In the middle of the crosswalk, there are two ways to set up safety island, namely, straight-through safety island and misaligned safety island. As shown in "Fig. 8", straightthrough safety island refers that the crosswalk and the safety island are on a straight line, and pedestrian and non-motor vehicle volume is small and applicable. The misaligned safety island refers that two crosswalks are separated in the middle, which increases the area of the safety island and provides enough safe stopping space.

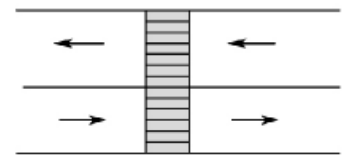

Straight-through Safety Island

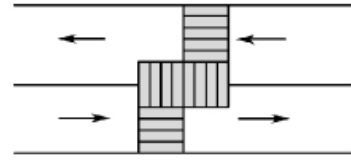

Misaligned Safety Island
Fig. 8. Setting method of safety island. 
3) Designing scheme for signal phases for twice crossing

There are two main ways to set up the signal for the pedestrian twice crossing: synchronous control and independent control. Independent control means that signal configuration at both ends of crosswalk is exactly the same, that is to say, the green light is turned on and off simultaneously. Independent control means that there is no necessary correlation between the green light on and off at both ends of crosswalk, which provides greater freedom of setting for the scientific allocation of pedestrian crossing time.

For the situation of this intersection, independent control signal setting is adopted.

In the original intersection phase organization, pedestrians only get the right-of-way in east-west and south-north straight phrases. After redesigning, the signal phase of pedestrian twice crossing is shown in "Fig. 9."

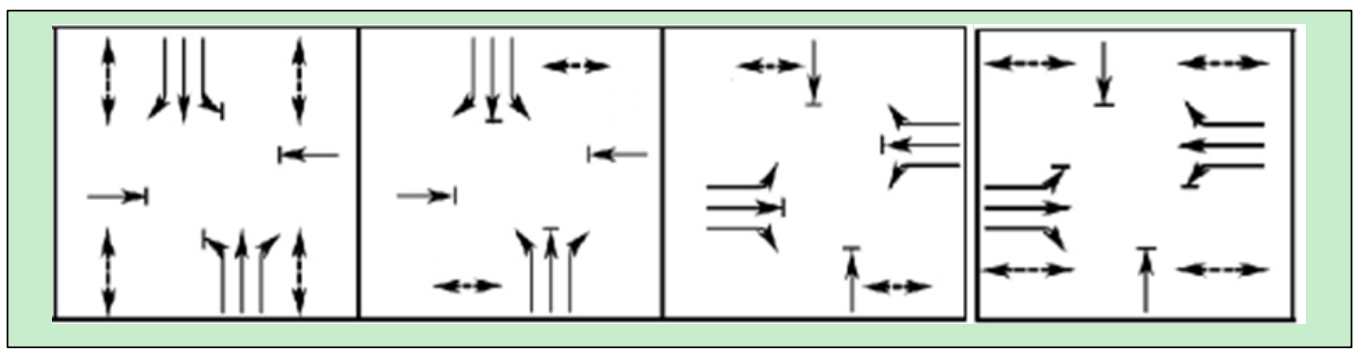

Fig. 9. Signal phase of pedestrian twice crossing after the design

In terms of the redesigned timing, when the signal control of crossing the street on the pedestrian crossing towards the North indicates turning left into the south and north, or east and west, the once crossing will be allowed in the twice crossing, that is, $36 \mathrm{~s}$ and $35 \mathrm{~s}$ is added to the time of crossing the street separately in this phase. The delay in crossing of pedestrian walking towards south is mainly due to the short green time of pedestrian crossing, so the south-north straight time will be extended to $38 \mathrm{~s}$. The volume of south-north leftturn vehicles is not large, so green time will be reduced to 31s. At the same time, it can also meet the time for pedestrians to reach the safety island at one time.

\section{INTERSECTION SIMULATION BASED ON VISSIM}

VISSIM software is used to simulate the current situation of the intersection and the situation after setting up the twice crossing, and the simulated data at peak time is shown in Table 3. It can be seen that the setting of twice crossing greatly reduces the delay of pedestrians and cars and improves the traffic capacity at the intersection.

TABLE III. COMPARISON OF SOME DATA IN PEAK HOURS BEFORE AND AFTER SETTING UP CROSSING FACILITIES

\begin{tabular}{|c|l|l|l|}
\hline $\begin{array}{c}\text { Crossing } \\
\text { Facility } \\
\text { Setting }\end{array}$ & $\begin{array}{c}\text { Pedestrian } \\
\text { Average } \\
\text { Crossing } \\
\text { Time/S }\end{array}$ & $\begin{array}{c}\text { Average / } \\
\text { Maximum } \\
\text { Waiting Number } \\
\text { / Person (One } \\
\text { Way) }\end{array}$ & $\begin{array}{l}\text { East exit road } \\
\text { turn right car } \\
\text { average delay } \\
\text { time/s }\end{array}$ \\
\hline Once Crossing & 52 & $35 / 45$ & 20 \\
\hline Twice Crossing & 35 & $26 / 34$ & 6 \\
\hline
\end{tabular}

\section{CONCLUSION}

Pedestrian is an important part of the pedestrian -vehicle conflict system, and the decreased operating efficiency will cause that the entire traffic system capacity cannot be effectively utilized. Especially at large signal-controlled intersections, pedestrians cannot cross the street at the green light signal once, resulting in conflict between pedestrians and motor vehicles and the decrease of pedestrian crossing efficiency and safety. Based on the analysis and study of traffic situation at the intersection, this paper analyzes the pedestrian crossing volume and the delay of right-turn cays in the east exit lane. After demonstrating the feasibility of the twice crossing, this paper proposes that when crosswalks are equipped with twice crossing and phase timing, applies vissim to simulate situation to prove the effectiveness of twice crossing, which can effectively utilize the space-time resources at signalized intersection, solve the traffic congestion caused by traffic conflicts at intersections and improve the crossing level at intersections.

\section{REFERENCES}

[1] Jennie Oxley,Rrian Fildes,Elfriede Ihsen,Judith Charlton,Ross Day. Differences in traffic judgments between young and old pedestrians.Acid.Anal,and Prev.29(6).1997.839-847.

[2] Tarawneh M S. Evaluation of pedestrian speed in Jordan with investigation of some contributing factors[J].Journal of Safety Research,2001,32(2):229-236.

[3] Knoblauch Richard,Crigler Kristy L. Model pedestrian safety program user's guide supplement [R]. Federal Highway Administration,1987.

[4] Yang Xiaoguang, Fu Qiang, Yang Xiaoguang. Setting Criterion of Pedestrian Twice Crossing at Signalized Control Link [J]. China Journal of Highway and Transport, 2006,19 (4): 109-113.

[5] Yang Xiaoguang, Lao Yunteng, Yun Meiping. Application of Different Pedestrian Cross Pattern to No-Signal Controlled Segment [J]. Journal of Tongji University (Natural Science), 2007, 35 (11): 104-108.

[6] Yang Rui. A Study on Pedestrian Delay Based on the Method of Pedestrian Twice Crossing at Signalized Intersection [D]. Southwest Jiaotong University, 2017.

[7] DGJ 08-96-2001, Design Regulations for At-grade Intersections on Urban Street [S].

[8] Martin A.Factors influencing pedestrian safety:a literature review[J]. Literature Review, 2006.

[9] Xiong Hui, Guo Hongwei, Lv Jian. Disaggregate Model for the Preference of Pedestrian's Crossing Facilities Selection [J]. Transactions of Beijing Institute of Technology, 2008, 28 (1): 37-40.

[10] GB 5020 - 1995, Code for Transport Planning on Urban Road [S].

[11] DGJ 08 - 96-2013, Shanghai Construction Standard - Design Regulations for At-grade Intersections on Urban Street [S]. 